\title{
The prospect of the dalton in the new SI: an educator's point of view
}

\author{
A. Barański
}

Received: 16 February 2013/Accepted: 19 July 2013/Published online: 8 August 2013

(C) The Author(s) 2013. This article is published with open access at Springerlink.com

\begin{abstract}
If the definitions of the kilogram and the mole, based on exact values of the Planck and Avogadro constants, respectively, are accepted within the framework of the new SI, then the current definition of the dalton cannot be retained. Acceptance implies redefinition of the dalton exactly in terms of the kilogram. The redefined, exact dalton is useless in mass spectrometry, and hence, a new quantity for the carbon-12 reference mass would have to be established-against the principle of Ockham's razor. In order to remove the roots of this awkward concept, the kilogram based on the Planck constant, and the mole, consisting of a particular number of entities equal to the inexactly determined numerical value of gram-to-dalton mass ratio, should be included in the new SI system. Some controversies related to the concept of mole have been also briefly outlined.
\end{abstract}

Keywords Dalton · New SI · Mole definition ·

Mole controversies · Avogadro constant

\section{Introduction}

This paper stems from two others. In an easily readable editorial, De Bièvre [1] has emphasized the importance of a

Papers published in this section do not necessarily reflect the opinion of the Editors, the Editorial Board, and the Publisher.

A critical and constructive debate in the Discussion Forum or a Letter to the Editor is strongly encouraged!

A. Barański $(\square)$

Faculty of Chemistry, Jagiellonian University, Ingardena 3, 30-060 Kraków, Poland

e-mail: baranski@chemia.uj.edu.pl measurable mass ratio, which is a number, for a description of chemical phenomena. In a metrological paper, Leonard [2] has revealed that an independent definition of the kilogram and the mole, as proposed by new SI, creates a fundamental incompatibility in stoichiometry if the dalton is still defined as $1 / 12$ th of the carbon-12 mass. The new SI concept of the mole should be broadly discussed by chemists [1]. This call has been backed by Milton's observation "there is very little initiative for ... a change from any of the communities of users of the mole" [3]. Another call by the BIPM has been addressed to the wide "scientific and user communities" [4]. But the comprehension of metrological concepts by an average user is not an easy task. This is why a simple and short paper is needed that presents a bird's eye point of view on the matter in question, as indicated by the title above. I do hope it will stimulate further discussion.

For the sake of easier presentation, two equations used by Leonard [2] have been rewritten below (see [2], Eqs. 5 and 6):

$1 \mathrm{~mol}=\frac{(\mathrm{g} / \mathrm{Da})}{N_{\mathrm{A}}}$ exactly

This fundamental definition of the mole was the result of a consideration involving the total substance mass, the atomic mass, the relative atomic mass, and the Avogadro constant, $N_{A}$. Consequently the relation,

$\left\{N_{\mathrm{A}}\right\}_{\mathrm{mol}^{-1}}=\frac{(0.001 \mathrm{~kg})}{\mathrm{Da}}$ exactly

where the left-hand side representing the numerical value of the Avogadro constant expressed in the unit reciprocal mole is valid "irrespective of how the kilogram and dalton are themselves defined". 


\section{Assumptions and the specific aim}

Let us consider a simple chemical synthesis of a product $\mathrm{P}$ from reactants of kind $\mathrm{A}$ and of kind $\mathrm{B}$. Both reactants are pure substances, i.e., they are chemical compounds or elements. Non-stoichiometric compounds are excluded from consideration. This enables us to specify a number of identical entities, like atoms, molecules, and ions that comprise the system. The mass of each entity, in Da, the unit accepted for use with the SI [5], can be found or calculated from physicochemical tables. The product $\mathrm{P}$ is leaving the system immediately. Diffusional obstacles are ignored. The stoichiometric equation of the synthesis is known.

The aim is to establish a criterion for masses of A and B that ensures the presence of both reactants, below the detection limit, after the reaction is completed in a laboratory or on an industrial scale. This is a trivial task today. However, in the nineteenth century, the above-outlined problem, being the implication of the law of definite proportions (Joseph Proust, 1797), was of pivotal importance for the development of chemistry and chemical technology.

\section{Avogadro's number}

\section{Atomic-scale description}

The chemical equation of the said reaction is known:

$a \mathrm{~A}+b \mathrm{~B} \rightarrow \mathrm{P}$

where $\mathrm{A}$ and $\mathrm{B}$ are the symbols denoting atoms or molecules; $a$ and $b$ are small integers, usually. We learn that $a$ entities A react with $b$ entities $\mathrm{B}$. The masses of the both reactants on the atomic scale, $m_{\mathrm{A}}$ and $m_{\mathrm{B}}$, are equal to $a M_{\mathrm{A}}$ and $b M_{\mathrm{B}}$, respectively. Here, $M_{i}$ denotes the atomic or molecular mass of an entity expressed in Da. The ratio of masses of both reactants is equal to

$\frac{m_{\mathrm{A}}}{m_{\mathrm{B}}}=\frac{a M_{\mathrm{A}}}{b M_{\mathrm{B}}}$

Equation (4) is a necessary and sufficient condition for the complete consumption of both reactants. However, the total mass of the system under consideration $\left(a M_{\mathrm{A}}+b M_{\mathrm{B}}\right)$ is very far from the laboratory and industrial conditions mentioned in the previous section. This is why in a conceptual experiment the mass of the system must be enlarged drastically.

Macroscopic-scale description and scaling factor

In the experiment in question, the masses of both reactants $\mathrm{A}$ and $\mathrm{B}, m_{\mathrm{A}}$ and $m_{\mathrm{B}}$, are multiplied by an ever-increasing number until the end of the experiment. At an advanced stage of this growth, when the values of the numbers are very high (of the order $10^{23}$ ), the atomic-scale unit, the dalton, is replaced by a macroscopic unit, the gram. When the mass values, expressed in $\mathrm{g}$, reach the initial mass values, expressed in Da, the further growth is discontinued. The system has reached its final macroscopic stage. The ratio of masses of both reactants, the same as in the Eq. (4) [1], implies the total consumption of the reactants during the reaction that would be completed on macroscale.

Now, we can calculate the scaling factor, commonly named Avogadro's number, $N_{\text {Avo, }}$, between the macroscopic-scale mass values measured in $\mathrm{g}$ and the atomicscale mass values expressed in Da. Consider the reactant A.

$N_{\mathrm{Avo}}=\frac{a M_{\mathrm{A}}(\text { in } \mathrm{g})}{a M_{\mathrm{A}}(\text { in } \mathrm{Da})}=1 \frac{\mathrm{g}}{\mathrm{Da}}$

That was indicated earlier [6]. The scaling factor $N_{\text {Avo }}$, as the ratio of two mass units, must be determined experimentally, i.e., $g$ must be expressed in Da. The dimensionless number thus obtained is $6.02214 \mathrm{X} \times 10^{23}$, where $X$ denotes the values of further digits being still to be determined [7]. If in Eq. (5) the gram were to be replaced, for example, by the pound or the tonne, $N_{\text {Avo }}$ would have another value. The principle of the determination of Avogadro's number, easy to comprehend, was briefly outlined in [8].

\section{The mole}

\section{Definition}

For the microscopic characterization of the system, it is necessary to estimate the number of entities that comprise it. However, a number of entities corresponding to a mass of the order of milligrams or more are needed in macroscopic experiments. This is a huge number, larger than $10^{20}$. Hence, counting atoms or molecules, as single entities, is impossible. Undoubtedly, a larger unit is needed. For its definition, Eq. (5) is useful.

The mole, symbol mol, is a particular number of entities, where that number is the gram/dalton mass ratio called Avogadro's number.

In a non-reacting system, the total number of entities, $N$, is proportional to the amount of substance, $n$, obviously. The proportionality factor, $N_{\mathrm{A}}$, is the Avogadro constant, and its unit is equal to $\mathrm{mol}^{-1}$, i.e., reciprocal moles.

$N=N_{\mathrm{A}} n$

Equation (6) is valid for all types of entities. By virtue of the definition of the mole, the numerical value of the 
Avogadro constant, expressed in reciprocal moles, is equal to Avogadro's number.

$\left\{N_{\mathrm{A}}\right\}_{\mathrm{mol}^{-1}}=N_{\text {Avo }}$

Note please the consistency of Eqs. (1) and (2), taken from [2], with Eqs. (5 and 7), seen in this paper.

Amount of substance and other controversial issues

Amount of substance is the official name of the quantity, represented by $n$, for which the mole is the SI unit. For decades, there has been endless debate concerning this quantity. For the sake of clarity, I will try to identify some main axes of the dispute, although, of course, they are interrelated with each other.

Some authors, e.g. [9], claim that mole is a macroscopic unit, whereas others, for example [10], emphasize its link with the number of entities on the atomic scale. In the twenty-first century, it is trivial to say that the description of a macroscopic system (consisting of atoms or molecules) can be done, in a macroscopic or in a microscopic way, by the same quantity. The equilibrium constant and the heat capacities expressed in terms of classical thermodynamics and statistical thermodynamics are the adequate examples here [11]. The quantity 'amount of substance' and its unit 'mole' are a similar case.

Both the macroscopic and the microscopic description are correct and are complementary to each other. A choice between them is determined by the issue under consideration. As the history of science is passing by, the microscopic or molecular approach is attracting more and more attention over the macroscopic or phenomenological approach because the former is able to reveal mechanisms of phenomena. The mole as the unit of amount of substance is a heritage of nineteenth-century phenomenological chemistry. At that time, it was the only choice because atoms had been considered as hypothetical entities only. The mole as the unit of an aggregate of entities, as proposed by Leonard [12], or an ensemble of entities, as proposed by Milton [13], fits well with scientific and educational concepts of the twenty-first century. For teaching purposes, the molecular approach is also the easier one, and this is why it is preferred here.

Two questions are closely related to each other. Is counting a measurement? Is it possible to define the mole as a number? The positive answer to the first question seems to be acceptable, especially since the publication of the paper entitled "Uncertainty in measurements by counting" [14]. Please note that in contrast to the second question, no formal decisions are needed in the first case. In principle, the answer to the second question is also "yes"-although without recommendation [12]. In addition, Leonard has shown [15] in detail, using the perfect gas law as the example, the modifications introduced to the equations of physical chemistry by the concept of a 'dimensionless mole'. The concept in question would also imply a redesigning of the SI that in turn would create the need of formal acceptance by the proper organizations under the Metre Convention. Note that in the more general case of 'units for quantities of dimension one' [16], the acceptance has not been granted.

There are claims that the mole and the kilogram, when expressed as a number of entities or a number of atomic masses, respectively, have to involve integers. This commonsense concept [17], when properly commented, may be helpful for teaching of chemistry, especially at the very early stage. However, the ratio gram/dalton is of order $10^{23}$, and usually, no more than 10 digits can be identified by an experiment-hence, the concept is insignificant.

Definitions of mole and dalton in the current SI and in the new SI

Let me emphasize a link between the final conclusions of the paper [2] and the ratio $\mathrm{g} / \mathrm{Da}=N_{\mathrm{Avo}}$ (see Eq. 5). As Leonard pointed out [6] any of two of $N_{\mathrm{Avo}}$, g and Da may be defined independently; then, the third is determined by Eq. (5).

The current concept of the mole has been determined by two definitions:

(1) The mass of the international prototype of kilogram (IPK) is greater than the mass of gram by the factor of $10^{3}$.

(2) The dalton is equal to $1 / 12$ th of the mass of the most abundant carbon isotope, namely carbon- 12 .

These definitions imply that Avogadro's number has to be determined by experiment. The same will be true if the IPK is replaced by the kilogram defined in terms of the Planck constant, commonly known as the 'electronic kilogram' ("The kilogram, $\mathrm{kg}$, is the unit of mass; its magnitude is set by fixing the numerical value of the Planck constant to be equal to exactly $6.62606 \mathrm{X} \times 10^{-34}$ when it is expressed in the unit $\mathrm{s}^{-1} \mathrm{~m}^{2} \mathrm{~kg}$, which is equal to J s". [7]).

However, the draft of new SI, endorsed already by CGPM [4], introduces simultaneously two exact values: for the Avogadro constant, when expressed in reciprocal mole, and for the 'electronic kilogram'. Hence, the third independent definition, namely the current definition of the dalton, cannot be retained. Experimental determination of the dalton is also excluded as the exact value of the Avogadro constant is already the result of the measurements linking the gram to the dalton. In this situation, in order to save the compatibility condition, the only choice is to calculate the dalton from the rearranged Eq. (5) [2]. 
$\mathrm{Da}=\frac{1}{1000\left\{N_{\mathrm{A}}\right\}_{\mathrm{mol}^{-1}}} \mathrm{~kg} \quad$ exactly

However, this redefined and exact dalton used in stoichiometry calculations is useless in mass spectrometry. Hence, a new inexact quantity, namely carbon- 12 reference mass, should be established for spectrometry purposes. This is against the principle of Ockham's razor rule, and conceptually very unfavorable, and unacceptable from the educational point of view, although very small differences between both quantities will yield no harmful practical implications,

\section{Final remarks}

The conceptual experiment described in this text is de facto an application of the molar mass constant in a descriptive way. Thus, the link of Avogadro's number with the inexactly known gram/dalton mass ratio is made easy to comprehend by students. This is a prelude to a definition of the mole. Note that the new SI is not necessary while introducing the definition that expresses the mole directly as a certain number of entities [8], at least in teaching. The renaming of 'amount of substance' is a similar case. All reasons summarized by Chyla [18] will be valid independently, whether the new SI is accepted or not.

What follows is my personal point of view on the SI reform. Let me balance the main advantages and the main drawbacks of the proposed new SI. The removal of the IPK, a decrease in uncertainty for some fundamental constants and satisfaction of the "electromagnetic community", caused by the appearance of the "electronic kilogram', seems to be the main advantages [19]. Decoupling of the dalton from the carbon-12 atomic mass standard [2], i.e., in fact, the creation of two 'daltons', that have exact and inexact values, respectively, is not acceptable. Teaching/learning difficulties related to the 'electronic kilogram' will create also a series of essential troubles.

Let me conclude. It seems that the optimal, new SI option is as follows: introducing the 'electronic kilogram' and retaining Avogadro's number as an inexact, experimental result. This will remove the roots of the troubles because no changes concerning dalton will be needed. In this context, let me quote the phrase by Guggenheim [20]. This will be "a tremendous triumph of reasonableness over confusion".

The future fate of the mole is clearly seen by Leonard, as outlined by the title of [12], and described also in the document [21]. I do hope the author will publish it soon. Articles should also be solicited describing how to teach the 'electronic kilogram'-from the elementary school up to the university level.

Acknowledgments Stimulating discussions with Professor Paul De Bièvre as well as valuable and inspiring comments of the referees are highly appreciated. I am also very grateful to professor Ian Mills for sharing his PowerPoint presentation from the CPEM meeting, Washington, July 2012, as well as to Professor B P Leonard for sharing his unpublished notes.

Open Access This article is distributed under the terms of the Creative Commons Attribution License which permits any use, distribution, and reproduction in any medium, provided the original author(s) and the source are credited.

\section{References}

1. De Bièvre P (2011) Integer numbers and their ratios are key concepts in describing the interactions of atoms and molecules. Accred Qual Assur 16:117-120

2. Leonard BP (2012) Why the Dalton should be redefined exactly in terms of the kilogram. Metrologia 49:487-491

3. Milton MJT (2011) A new definition for the mole based on the Avogadro constant: a journey from physics to chemistry. Phil Trans R Soc A 369:3993-4003

4. BIPM (2012) On the possible future revision of the International System of Units, the SI. http://www.bipm.org/utils/en/pdf/24 CGPM_Resolution_1.pdf. Accessed 13 Jun 2013

5. BIPM (2006) The International System of Units, 8th edn. Bureau International des Poids et Mesures, Sèvres, France, p 126

6. Leonard BP (2010) Comments on the recent proposals for redefining the mole and kilogram. Metrologia 47:L5-L8

7. Mills IM, Quinn TJ, Taylor BN (2010) Draft chapter 2 for SI Brochure, following redefinitions of the base units. http://www. bipm.org/utils/common/pdf/si_brochure_draft_ch2.pdf Accessed 13 Jun 2013

8. Barański A (2012) The atomic mass unit, the Avogadro constant, and the mole: a way to understanding. J Chem Educ 89:97-102

9. Wheatley N (2011) A sorites paradox in the conventional definition of amount of substance. Metrologia 48:L17-L21

10. Price G (2011) A skeptic's review of the New SI. Accred Qual Assur 16:121-132

11. Atkins P, de Paula J (2006) Atkins' Physical Chemistry 8th ed. Oxford Univ. Press, chap. 2 and 7 (classical thermodynamics), chap.17 (statistical thermodynamics)

12. Leonard BP (2007) The atomic-scale unit, entity: key to a direct and easily understood definition of the SI base unit for amount of substance. Metrologia 44:402-406

13. Milton MJT (2013) The mole, amount of substance and primary methods. Metrologia 50:158-163

14. Bich W, Pennecchi F (2012) Uncertainty in measurements by counting. Metrologia 49:15-19

15. Leonard BP (2011) Alternative interpretations of the mole and the ideal gas equation. Accred Qual Assur 16:577-581

16. Dybkaer R (2004) Units for quantities of dimension one. Metrologia 41:69-73

17. Hill TP, Miller J, Censullo AC (2011) Towards a better definition of the kilogram. Metrologia 48:83-86

18. Chyla WT (2012) On the proposed redefinition of mole. Metrologia 49:L11-L13 sect. 5

19. Mills I (2012) Adapting the international systems of units to the 21 st Century-powerpoint presentation of the lecture during the 
conference "precision electromagnetic measurements" Washington, DC, USA; 1-6 July

20. Guggenheim EA (1961) The mole and related quantities. J Chem Edu 38:86-87
21. Leonard BP (2013) A tale of two Avogadro numbers, The unpublished notes prepared as a document for attendees of the 19th meeting of the CCQM, April 12-19, 2013; received due to courtesy of the author 